\title{
Deconstructing the mental health crisis in only 2 pieces
}

\author{
Ridha Joober, MD, PhD
}

In their editorial in this issue of JPN, Merali and Anisman ${ }^{1}$ proposed a deconstruction of the mental health crisis in "5 uneasy pieces." While a crisis may exist, there may be divergence on how to define this crisis, how it can be deconstructed and how we can move beyond it.

First, from Merali and Anisman's use of the term "treatment" almost always being synonymous with pharmacological treatment, their quasi-silence on nonpharmacological treatments and from the type of research they are promoting, it can be deduced that the crisis the authors described is mainly one of biological psychiatry. Second, there is certainly a crisis in mental health, which encompasses more than biological psychiatry, but I believe that the nature of the 2 crises and the responses that they require may be very different and probably at times antagonistic. So I propose to deconstruct the crisis into 2 pieces: biological psychiatry and mental health.

\section{The crisis in biological psychiatry}

Merali and Anisman present 3 key points on the crisis in biological psychiatry: big pharmaceutical companies are withdrawing from the field, advanced technologies have failed to provide any clinical utility in psychiatry, and very little progress has been made in identifying biomarkers for mental disorders. What also adds to the evidence of this crisis is the contrast with the rest of the medical domains, where important advances in drug discovery, diagnostics and biomarkers have been made. Indeed, one can only wonder how little biological psychiatry has done in the field of therapeutics since the initial serendipitous discovery of the major classes of psychotropic medications. Certainly, the safety and tolerability profiles of many classes of psychotropics have been improved, but no new biological targets or more efficacious medications have been identified despite tremendous efforts deployed and billions of dollars spent. To cite 1 example, the most effective medications in modifying behaviours, methylphenidate and amphetamine, which are used for the treatment of attention-deficit/hyperactivity disorder, have been around for more than 70 years and have yet to be surpassed. Thousands of brain imaging studies (including studies originating from my own research group) have failed to go beyond the now trivial report of statistical group differences between patients and controls with regard to some indices in brain parameters. It might be easy to say that something will happen if we keep enlarging our networks and waiting for the next new technology; however, after decades of this attitude persisting, the industry, the funding agencies, the philanthropists, the public in general, a large contingent of scientists working in the field and, most importantly, patients and their families are losing faith.

According to the renowned philosopher of science Thomas Kuhn, a crisis in a specific domain of science arises when the scientific community starts to lose faith in their paradigm. Is the field of biological psychiatry in crisis? The question is open, but there are strong and robust indicators that this might indeed be the case. One of these indicators is the loss of faith of many, as Merali and Anisman alluded to in their editorial. But most importantly, there are findings and attitudes in biological psychiatry that point to the deep schism between biological psychiatry and mental health. Most of the psychiatric disorders have very strong genetic underpinnings, and biological psychiatry has done an outstanding job identifying the genetic variants increasing the risk for these disorders: thousands of genetic variations, each with a very small effect size, are at play. The "dusty" nature of these effects seem to be more true for highly prevalent disorders those with the highest cost for society, like depression and anxiety. To give a recent example, a large meta-analysis of genome-wide association studies in neuroticism and depression identified a single highly significant but weak (odds ratio $=1.04$ ) association with a single nucleotide polymorphism using a sample of more than 63000 individuals! This result could not be replicated in a sample of 9786 individuals. ${ }^{2}$ The question is how can we act on this knowledge? Optimists would say we will do something and rush to press releases. However, it is sobering to remind ourselves how the community of biological psychiatry has failed in the past to

Correspondence to: R. Joober, Douglas Mental health University Institute and McGill University, 6875 Boulevard LaSalle, Montreal H4H 1R3; ridha.joober@douglas.mcgill.ca

DOI: 10.1503/jpn.160103 
act on much stronger associations. Because suicide may be a very good sentinel indicator of how well psychiatry is contributing to improved mental health, and because unfortunately the news is not so good on this front, ${ }^{3}$ I will use 2 examples to illustrate this failure of action. The dexamethasone suppression test (DST) is a glaring example. Patients with depression and suicidal ideation who fail to suppress cortisol secretion after the DST have been shown to be 9.2 times more at risk for committing suicide in the next 15 years of their follow-up. ${ }^{4}$ This is equivalent to the association between smoking and lung cancer, but the cancer community and the public health authorities have acted and succeeded in reducing smoking and lung cancer. ${ }^{5}$ Nothing similar has been done as far as the DST and suicide is concerned. Another study identified a single biological marker that discriminated $100 \%$ of participants who where exposed to childhood trauma and who committed suicide from controls who did not commit suicide! ${ }^{6}$ Was there any practical follow-up study for this astonishing result? None! Why is that? A lot can be said about the failures to act on strong biological findings in psychiatry, but maybe this indicates poor faith in these results in our own biological psychiatry research community. What is more worrisome is that we turn a blind eye to these failures. While larger networks, better science and more money could help to advance biological psychiatry, it may be very helpful to ask ourselves hard questions about the role of biological psychiatry in mental health.

\section{Major facets in the mental health crisis}

What is the second crisis in mental health? To their credit, Merali and Anisman mentioned some of the elements of the crisis in mental health. Yes, mental disorders are very prevalent and very costly. Yes, stigma - not only self-stigma, but also stigma among the general public and health providers is rampant. The crisis in mental health also has other major facets. One of the most important, at least in Canada, is that psychological and psychosocial treatments are not fully recognized and compensated. Psychological approaches are effective but not supported by health care payers and are not featured prominently in curricula of mental health professionals, particularly psychiatrists. Even pharmacological treatments that are effective are not used as they should be. Clozapine, for example, a treatment that has been shown time and time again to be the only medication effective in treating resistant schizophrenia, is very much underused.7 The organization of services remains fragmented, and pathways to care are tortuous and contribute strongly to delayed care and disengagement from services and treatment, particularly among youth. ${ }^{8}$

Another determinant of the mental health crisis is its failure to act on many of its strongest findings. For example, child abuse and maltreatment, which are also very prevalent, have been shown to be associated with major depression, anxiety and psychosis. It is unclear how much mental health professionals are engaged with the public health authorities to prevent childhood abuse and maltreatment.

Finally, it could be argued that biological psychiatry has strongly contributed to the crisis in mental health, by its strong emphasis on biology and the promotion of a simplistic model of the association between the brain and mental illnesses. The industry, with its powerful marketing force has strongly promoted the "pill solution," despite the absence of innovation. Public funders, philanthropists and journalists are easily tempted by the idea that there will be a pill to treat each mental disorder, and the patients have also been conditioned to believe that this might be the case, which results in questionable attitudes and expectations.

Fortunately, on this crisis front, we have seen some comforting efforts to fund research directed toward a more integrated view of mental health care in the last 10 years. For example, the Mental Health Commission of Canada (MHCC) has funded the project "Chez Soi" to investigate the effects of supported housing on mental health. The Canadian Institutes of Health Research (CIHR) Strategy for Patient-Oriented Research (SPOR) initiative in conjunction with the Graham Boeckh Foundation recently funded a project to transform youth mental health services in Canada (ACCESS-Open minds). While these and many others are promising good indicators, much more needs to be done.

\section{Mental disorders are more than brain disorders}

The main paradigm of biological psychiatry is that "mental disorders are brain disorders." But how we go about truly understanding this statement can make a huge difference in how we work. The dominant understanding of this paradigm follows the medical model: once the organ responsible for the disease is identified, we need to find out what is wrong in the working of that organ and subsequently fix the dysfunction to cure the disease. For psychiatric disorders, the brain is certainly the organ in question (this was established hundreds, if not thousands, of years ago), but unlike all other somatic illnesses, the nature of the dysfunction leading to disordered minds turned out to be very difficult to pinpoint. The biological psychiatry crisis may be due to an inadequate extrapolation of the medical model to the brain and mind. To draw a parallel that will help to clarify the inadequacy of this extrapolation, it can be very firmly stated that molecular biology (MB) is rooted in quantum mechanics (QM). Yet, to my knowledge, no textbook has tried to propose understanding MB on the basis of QM. This may be because these 2 fields of science, although the former is entirely rooted in the latter, operate with different paradigms and experimental approaches. Most importantly, we can understand each of them and make tremendous advances without necessarily being fixated on how QM explains MB. Coming back to biological psychiatry, it is quite easy to accept that the distance separating the brain and mind is immense compared with the one that separates QM and $\mathrm{MB}$ and that mental health can progress and thrive without being heavily dependent on biological psychiatry. I believe that after the serendipitous discovery of psychotropic medication early in the last century, biological psychiatry has contributed greatly to psychiatry, not so much by showing that any molecular or brain anomaly has any clinical utility for psychiatric practice, but by ushering the need for a renewed 
paradigm in biological psychiatry: mental disorders are much more than brain disorders!

So how can biological psychiatry contribute to mental health? I believe that biological psychiatry is one of the major models helping us to chaperone our thinking and to develop part of the narrative that we use to interact with our patients and their families in their quest (and ours) to understand their mental states and how therapies, pharmacological or otherwise, can help them improve their mental states. Presenting refined biological models (along with other models), while at the same time recognizing their strong limitations when applied to individual situations, is an important part of our mission and of the healing process. I also believe that biological psychiatry, presented in this renewed paradigm, can help us to educate future generations of scientists and mental health professionals, the media, funding agencies and the public about the complexity of human behaviours and the privileged place of the individual, aided by different approaches, as the central agent of change. Redefining the goals of biological psychiatry, setting the right expectations and reasonable timelines would be essential for biological psychiatry to continue its slow, but fundamental contribution. But I would be delighted to be proven wrong about the slow pace, tomorrow!

Affiliations: From the Douglas Mental health University Institute and McGill University, Montreal, Que., Canada.
Competing interests: R. Joober sits on the advisory boards and speakers' bureaus of Pfizer Canada, Janssen Ortho Canada, BMS and Sunovian Canada; he has received grant funding from them and from AstraZeneca. He has received honoraria from Janssen Ortho Canada and from Pfizer Canada for CME presentations and royalties for Henry Stewart talks.

\section{References:}

1. Merali Z, Anisman H. Deconstructing the mental health crisis: 5 uneasy pieces. J Psychiatry Neurosci 2016;4:219-21.

2. Genetics of Personality Consortium, de Moor MH, van den Berg $\mathrm{SM}$, et al. Meta-analysis of genome-wide association studies for neuroticism, and the polygenic association with major depressive disorder. JAMA Psychiatry 2015;72:642-50.

3. Centers for Disease Control and Prevention. Increase in suicide in the united states, 1999-2014. Available: www.cdc.gov/nchs/products /databriefs/db241.htm (accessed 2016 June 2).

4. Coryell W, Schlesser M. The dexamethasone suppression test and suicide prediction. Am J Psychiatry 2001;158:748-53.

5. Pastorino U, Boffi R, Marchianò A, et al. Stopping smoking reduces mortality in low-dose computed tomography screening participants. J Thorac Oncol 2016;11:693-9.

6. McGowan PO, Sasaki A, Huang TC, et al. Promoter-wide hypermethylation of the ribosomal RNA gene promoter in the suicide brain. PLoS One 2008;3:e2085

7. Latimer E, Wynant W, Clark R, et al. Underprescribing of clozapine and unexplained variation in use across hospitals and regions in the Canadian province of Québec. Clin Schizophr Relat Psychoses 2013;7:33-41.

8. Norman RM, Malla AK, Verdi MB, et al. Understanding delay in treatment for first-episode psychosis. Psychol Med 2004;34:255-66.

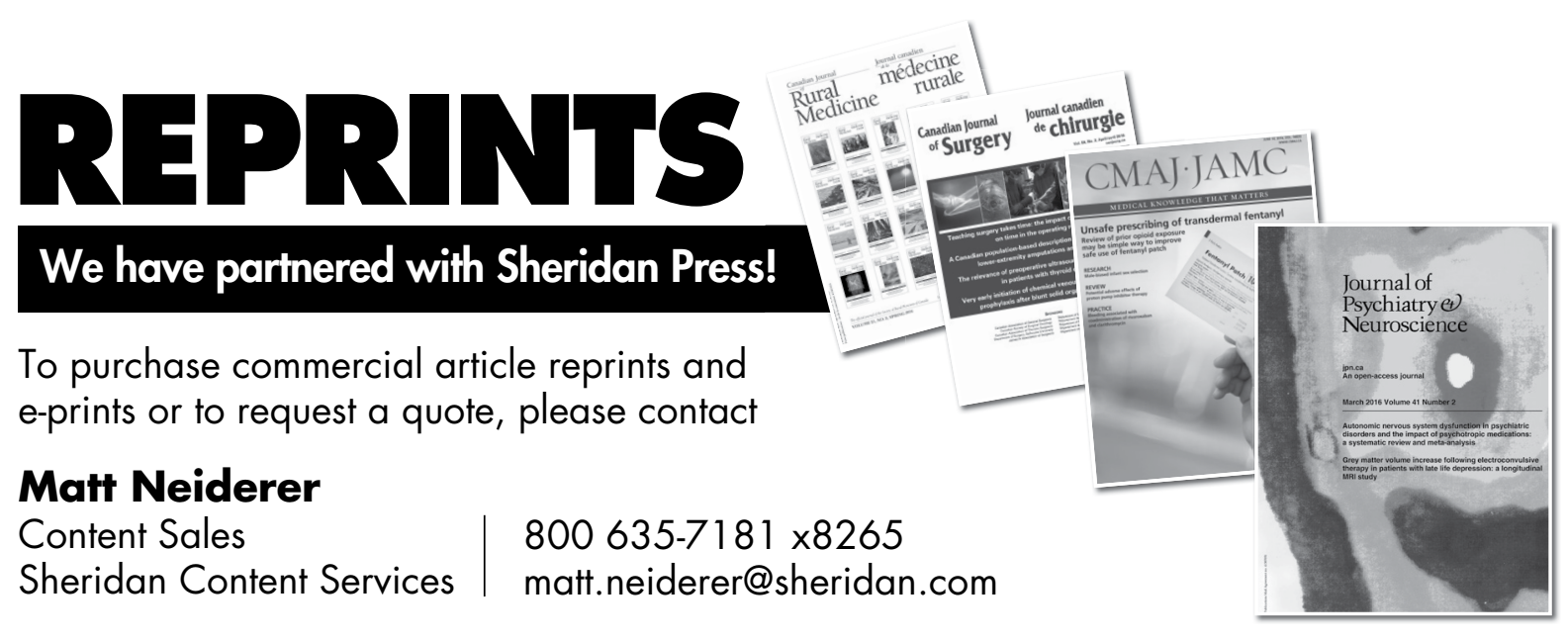

